\title{
Effect of Water Spray on Re-ignition Characteristics of Solid Fuels
}

\author{
B. MOGHTADERI, V. NOVOZHILOV and J.H. KENT \\ Department of Mechanical and Mechatronic Engineering \\ The University of Sydney, NSW 2006, Australia \\ D.F. FLETCHER \\ Department of Chemical Engineering \\ The University of Sydney \\ NSW 2006, Australia \\ V.B. APTE \\ Londonderry Occupational Safety Centre \\ 132 Londonderry Road \\ Londonderry, NSW 2753, Australia
}

\begin{abstract}
A set of small-scale experiments was carried out to study the effect of a water spray on the re-ignition characteristics of solid fuels. The influence of other key parameters, such as the incident heat flux and pre-burn, was also investigated. The experiments were conducted on specimens of wood and PMMA using a cone calorimeter. A water spray was produced by a small commercial nozzle. As expected, the effect of water on the re-ignition time was found to be significant. It was also found that the re-ignition characteristics of charring materials, such as wood, are quite different from non-charring materials (e.g. PMMA) mainly due to the structural differences. Based on the experimental observations a set of empirical correlations was obtained for both wood and PMMA samples. Predictions of the re-ignition time made by these correlations agree well with the measurements. Simulations of the re-ignition times were also performed using a detailed mathematical model. Comparisons with the experimental data are provided.
\end{abstract}

KEYWORDS: Re-ignition; solid fuels; water spray; cone calorimeter.

\section{NOMENCLATURE}

$c_{p} \quad$ Specific heat $\left(\mathrm{kJ} \mathrm{kg}^{-1} \mathrm{~K}^{-1}\right)$

$H_{v} \quad$ Heat of evaporation $\left(\mathrm{kJ} \mathrm{kg}^{-1}\right)$

$L \quad$ Sample thickness (m) 
$M_{0} \quad$ Initial sample weight $(\mathrm{kg})$

$m_{w} \quad$ Total water applied $(\mathrm{kg})$

$m_{w, c r}$ Critical value of the total water applied $(\mathrm{kg})$

$m_{c r}$ Critical mass flux of volatiles $\left(\mathrm{kg} \mathrm{s}^{-1} \mathrm{~m}^{-2}\right)$

$P B \quad$ Pre-burn

$q_{c}^{\prime \prime} \quad$ Heat loss due to cooling $\left(\mathrm{kW} \mathrm{m}^{-2}\right)$

$q_{e}^{\prime \prime} \quad$ External heat flux $\left(\mathrm{kW} \mathrm{m}^{-2}\right)$

$T \quad$ Temperature (K)

$t_{d} \quad$ Drying time (s)

$t_{i p b} \quad$ Ignition time of the partially burnt material (s)

$t_{r i} \quad$ Re-ignition time (s)

$t_{d}{ }^{*} \quad$ Characteristic drying time (s)

$t_{i p b}{ }^{*} \quad$ Characteristic ignition time (s)

$\alpha \quad$ Thermal diffusivity $\left(\mathrm{m}^{2} \mathrm{~s}^{-1}\right)$

$\delta \quad$ Thermal penetration depth (m)

\section{INTRODUCTION}

Fire suppression in buildings usually involves the extinguishment of fires from solid fuels. In such an environment, when a burning object is extinguished, it may still receive a substantial amount of radiative energy from other burning objects. As a result, the extinguished object may re-ignite. Here, re-ignition refers to the reappearance of a flame in the volatile gas stream evolved from the solid. Since water sprays are the most common method used in fire extinguishment [1,2], it is desirable to investigate their effects on the re-ignition phenomenon.

A literature survey shows that only a few scientific investigations dealing with the problem of re-ignition have ever been undertaken. Depew et al. [3] studied the response of a cellulosic object, exposed to external radiation, to the impingement of water droplets. They also quantified the recovery time of the surface which was defined as the time required for re-ignition under a constant radiative heat flux following a burst of water. However, no rational framework was established to quantify the effects of controlling factors, such as fuel type and amount of pre-burn. Besides this work was mainly concerned about the re-ignition phenomenon in wild land fires.

More recently, Usui [4] conducted a series of experiments on re-ignition phenomenon of wood cribs. Based on Usui's data Takahashi [5] obtained a simple theoretical formula which provides the relationship between the re-ignition time and the water content of the wood ember. This formula was then revised to fit a wider range of heating conditions [6-8]. None of these recent works have addressed the re-ignition of other types of fuels, such as plastics, or other geometrical configurations. Therefore, a more extensive database is necessary.

The present work constitutes part of a long-term study aimed at the modelling of fire spread and extinguishment in building fires. In particular, the research presented here investigates the re-ignition of extinguished solid fuels under constant external radiation. The main objectives here are: (a) to obtain a more fundamental and universal description of the re-ignition problem, and (b) to establish a database of re-ignition data of some common solid fuels as a function of applied water, pre-burn and incident flux. For this 
purpose a series of small-scale experiments were performed on specimens of wood and PMMA using a modified cone calorimeter. Analysis of the experimental data was performed to obtain useful and practical correlations. Experimental results were also compared with theoretical predictions obtained from a detailed mathematical model.

\section{EXPERIMENTAL}

\section{Materials}

Experiments were conducted on specimens of Radiata pine wood and PMMA. More than 100 samples were used for these experiments. All samples were $100 \mathrm{~mm}$ square as required for testing in the cone calorimeter. The thicknesses of PMMA and wood samples were 9 and $19 \mathrm{~mm}$, respectively. All samples were tested in the horizontal orientation.

The samples of Radiata pine were cut from one timber length to improve the consistency of tests. In order to eliminate the effect of moisture content, wood samples were also oven-dried at $105^{\circ} \mathrm{C}$ to a constant mass and then stored in a desiccator. The oven-dry densities of wood samples were found to be fairly constant with an average value of $465 \mathrm{~kg} / \mathrm{m}^{3}$.

\section{Apparatus and Procedure}

In this study, a modified version of the standard cone calorimeter (ASTM E 1354 [9]) was utilised. A schematic illustration of the experimental apparatus is shown in Figure 1. The water spray was applied manually from a nozzle assembly aimed at the fuel sample. A commercially available full-cone nozzle (BLM 4-90) with an effective angle of about $90^{\circ}$ was used to generate the water spray. The distance between the nozzle tip and the sample were kept at $7 \mathrm{~cm}$ to obtain full coverage of the sample surface. Water was filtered before it was fed to the nozzle. During each test the nozzle flow was held constant by maintaining a constant water pressure. The maximum operating pressure used in this study was $300 \mathrm{kPa}(\approx 3 \mathrm{~atm})$. No attempt was made to measure the drop size distribution. However, according to information supplied by the manufacturer, at an operating pressure of $300 \mathrm{kPa}$ the volume median diameter of the drops was 850 microns.

The rate of water application was varied by changing the operating pressure. Pressures below $200 \mathrm{kPa}$ were never used due to irregularities caused by low operating pressures. As a result, a narrow range of water flow rates $\left(0.25-0.30 \mathrm{kgm}^{-2} \mathrm{~s}^{-1}\right)$ was produced. (The chosen range of water flow rates is much higher than the minimum values required for the suppression of fires [10]. However, it is similar to water flow rates which are used in actual fire fighting practices [11]. In addition, for the present experimental setup, this range of water flow rates provides an instantaneous extinguishment which is highly desirable.) At each flow rate, the total amount of applied water was controlled by the application time which was varied from 5 to 15 (s). The actual rate of water application was determined by measuring the rate at which water accumulated on a nonburning sample. This value was assumed to be the same as that existing during the actual test.

The experimental procedure involved the following steps. At first, the radiant heater was set to the desired value. Prior to testing, all specimens were laid on Kaowool mat in a 


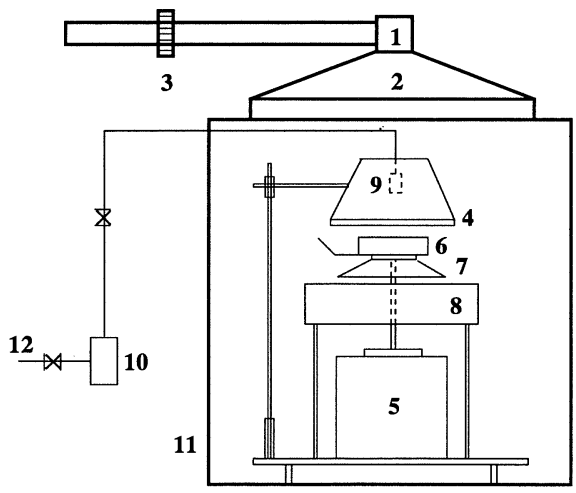

1. Exhaust Duct

2. Hood

3. Exhaust Fan

4. Radiant Heater

5. Load Cell

6. Sample Holder

7. Water-Proof Shield

8. Tray

9. Nozzle

10. Filter

11. Enclosure

12. Main Water Supply Line

FIGURE 1: Experimental Set-up

$25 \mathrm{~mm}$ deep stainless steel tray, such that the surface of the specimen was level with the top edge of the tray. The unexposed surface and edges of each specimen were wrapped in aluminium foil thereby minimising the edge effects and approaching the one-dimensional heat transfer case. The sample was then exposed to the external radiation by placing the sample holder under the radiant heater. No external ignition source was used, instead spontaneous ignition was allowed to occur. Once a stable flame appeared at the surface of the specimen, it was allowed to burn under the conditions selected until the sample weight diminished to a pre-determined weight. The application of the water spray was then started in order to extinguish the flame. At the same time measurement of the re-ignition time was started. The water spray was left on for a selected period of time $(5,10$ or $15 \mathrm{~s})$ which was much longer than the time required for extinguishment. The specimen was kept under the external radiation until the specimen re-ignited. Re-ignition was judged to have been reached when a stable flame reappeared at the sample surface. This procedure was repeated for various combinations of radiant flux, water application rate and pre-burn which is defined as: $\% P B=\left(M_{0}-M\right) / M_{0}$, where $M_{0}$ is the initial sample weight and $M$ is the weight at the beginning of water spray application.

\section{VISUAL OBSERVATIONS}

Visual observations reveal that the re-ignition time includes three sub-intervals corresponding to (a) extinguishment, (b) evaporation of water droplets, and (c) ignition of partially burnt material.

As Figure 2 illustrates, because of the high flow rates of water used in this study, the extinguishment times are quite short and scattered. Moreover, there is no apparent relationship between the time of extinguishment and key parameters, such as pre-burn and incident heat flux. It seems, however, that the extinguishment time decreases as the water flow rate increases.

The short extinguishment times observed in this study allow us to assume that the flame extinction is instantaneous. As a result, the extinguishment and evaporation subintervals can be treated as a single phase which is called here the drying phase. In this 


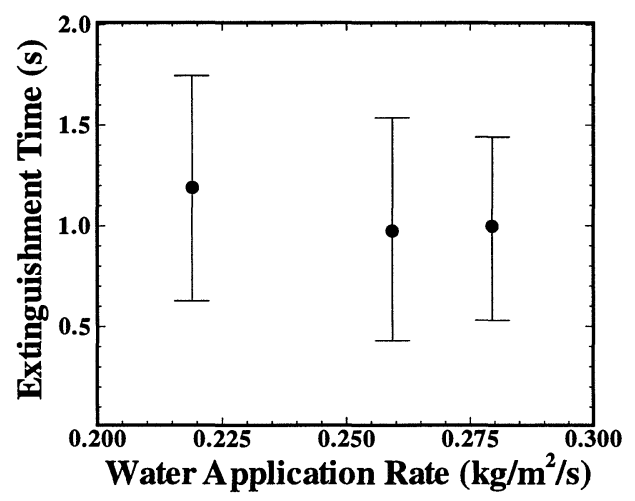

FIGURE 2: Experimental values of the extinguishment time. upon the surface. This corresponds to a spray film cooling mode [12]. Regardless of the fuel type, formation of the liquid layer was observed to occur between 4-5 (s) after the start of the spray application. Based on visual observations the size of the liquid layer depends on the water flow rate, the application time of the spray and fuel type. If this size becomes large enough, water will run-off from edges of the sample. For wood, run-off occurred at flow rates greater than $0.28 \mathrm{kgm}^{-2} \mathrm{~s}^{-1}$ and application times longer than $10(\mathrm{~s})$. For PMMA the corresponding values were $0.25 \mathrm{kgm}^{-2} \mathrm{~s}^{-1}$ and 10 (s), respectively.

Once the water spray is turned off, the size of the liquid layer begins to decrease under the influence of the external radiation. For wood specimens this is followed by evolution of steam from the fissures and cracks formed at the surface. The end of the drying phase for wood samples is marked when the flow of steam ends. However, for PMMA samples the disappearance of the liquid layer from the surface marks the end of the drying phase.

\section{EXPERIMENTAL RESULTS AND DISCUSSION}

Several types of results were obtained from each test including weight loss, drying time $\left(t_{d}\right)$, ignition time of the partially burnt material $\left(t_{i p b}\right)$ and re-ignition time $\left(t_{r i}\right)$. The relation between these different times can be expressed as: $t_{r i}=t_{d}+t_{i p b}$.

Typical sets of characteristic times $\left(t_{d}, t_{i p b}\right.$ and $\left.t_{r i}\right)$ are shown in Figures 3 and 4 for Radiata pine and PMMA at an incident heat flux of $25 \mathrm{~kW} / \mathrm{m}^{2}$ and $10 \%$ pre-burn. In these figures the total water applied $\left(m_{w}=\right.$ flow rate $\times$ spray application time) has been drawn, as the abscissa and the relevant time components along the ordinate axis.

Figures 3 and 4 indicate that for both materials $t_{i p b}$ is weakly dependent on $m_{w}$. As a result, the re-ignition time follows almost the same trend as $t_{d}$ (note that $t_{r i}=t_{d}+t_{i p b}$ ). Two distinct regions can be identified in each figure and a critical value of applied water $\left(m_{w, c r}\right)$ may be used as the boundary between these two regions. Based on the experimental results, values of $m_{w, c r}$ for Radiata pine and PMMA are 3.3 and $2.5 \mathrm{~kg} / \mathrm{m}^{2}$, respectively. As Figures 3 and 4 show, in the first region $\left(m_{w} \leq m_{w, c r}\right)$ water has a strong influence on $t_{d}$ and 


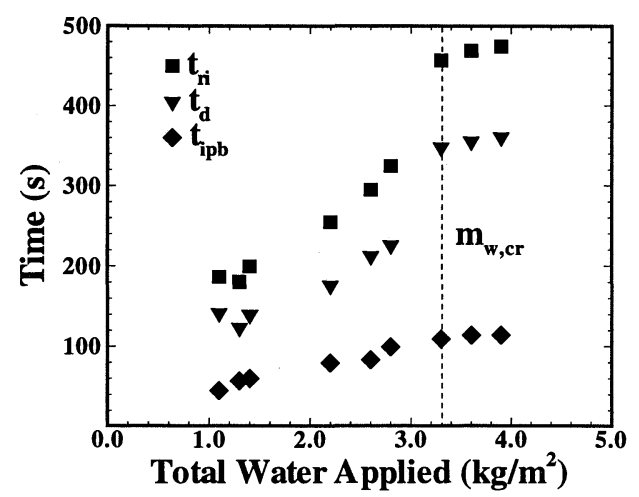

FIGURE 3: Measured characteristic times for Radiata pine at an incident heat flux of $25 \mathrm{~kW} / \mathrm{m}^{2}$ and $10 \%$ pre-burn.

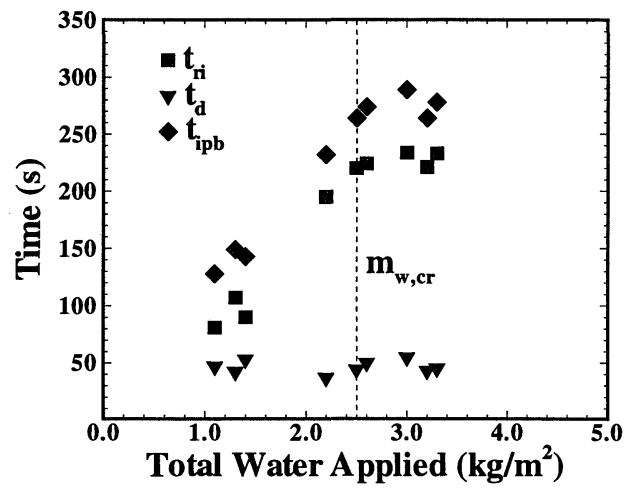

FIGURE 4: Measured characteristic times for PMMA at an incident heat flux of $25 \mathrm{~kW} / \mathrm{m}^{2}$ and $10 \%$ pre-burn. consequently $t_{r i}$. Despite the similarities, PMMA and wood exhibit different behaviours in this region. For PMMA, Figure 4 shows that $t_{d}$ and $t_{r i}$ have linear relationship with $m_{w}$, whereas for wood there is a nonlinear relationships (see Figure 3). The linear behaviour is expected because most of the heat required for evaporation of the liquid layer comes from the external radiation which is constant. However, the overall process may also be affected by the cooling of the solid which is another source of heat supply for the evaporation process.

The effect of cooling, in the case of PMMA, is not significant. However, wood like other natural solids is porous and produces a low thermal conductivity char layer during burning which is even more porous than the virgin material. When water is applied to such a porous structure the overall cooling process is greatly enhanced mainly due to the large contact area between the water and the hot char layer. As $m_{w}$ is increased the contact area and subsequently cooling rate are also increased, but there is a limit. Once all cavities at the surface are filled with water there is no more increase in the contact area. Therefore, the functional relationship between the cooling rate and the amount of applied water is not a simple function of $m_{w}$. That explains why wood shows a nonlinear behaviour in the first region.

As Figures 3 and 4 indicate, in the second region $\left(m_{w}>m_{w, c r}\right)$ the characteristic times are almost independent of $m_{w}$. The main reason for such behaviour is that as $m_{w}$ is increased beyond the critical value, water begins to run off the surface. Thus, the amount of water absorbed by the sample and consequently $t_{r i}, t_{d}$ and $t_{i p b}$ remain practically constant.

The effects of other controlling parameters on the re-ignition time are shown in Figure 5 , where $t_{d}$ has been plotted as a function of the incident heat flux and pre-burn. As shown in this figure, the re-ignition time for Radiata pine is generally in the range of 1 to 8.5 minutes. Also the sets of data for each incident heat flux collapse onto a separate surface. The effect of pre-burn is evident. The higher the pre-burn the shorter the reignition time. This is quite obvious because in order to increase $\% P B$ the solid must be 


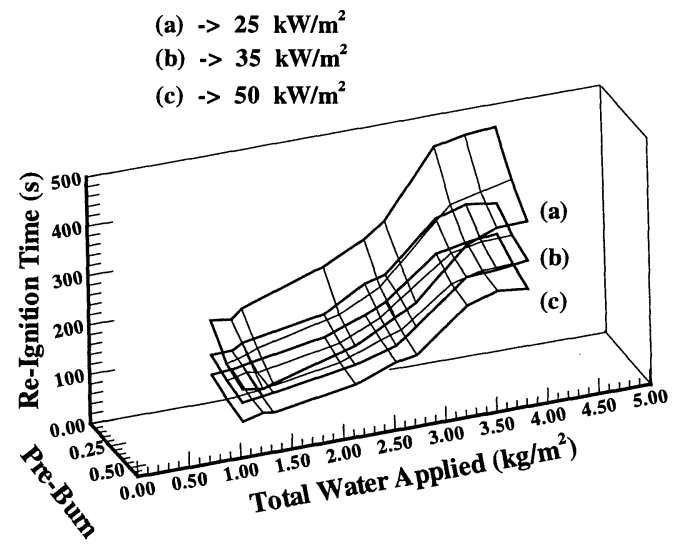

FIGURE 5: Measurements of the re-ignition time for Radiata pine.

\section{CORRELATION OF EXPERIMENTAL RESULTS}

The measured re-ignition times for PMMA and wood can be correlated in terms of $t_{d}$ and $t_{i p b}$. Analysis of experimental results revealed that for both materials, the product of the drying time by incident heat flux $\left(q_{e} /\right)$ is a variable which weakly depends on the incident heat flux. This variable which is called here: Flux Time Product (FTP) may be used to correlate the measured drying times. As a result, the functional relationship between $t_{d}$ and controlling parameters is expressed as: $t_{d} \propto f\left(m_{w}, \% P B\right) / q_{e}^{\prime \prime}$, where the function $f$ can be obtained from the curve fits of FTP. Also the experimental data of $t_{i p b}$ may be correlated using the relation: $t_{i p b} \propto g\left(q_{e}\right.$, $\left.\% P B\right)$, where $g$ is only a function of incident heat flux and pre-burn. This is a reasonable assumption because according to the present set of data, $m_{w}$ does not have a strong influence on $t_{i p b}$. Based on the procedure outlined here, the following correlations are proposed:

(a) Wood

$$
\begin{aligned}
& t_{d}=\left(-\left(9.4 \times 10^{3}+50.5 \times \% P B\right)+\left(2.8 \times 10^{4}+52.5 \times \% P B\right) m_{w}-\left(2.2 \times 10^{2}+22.4 \times \% P B\right) m_{w}{ }^{2}\right. \\
& \left.+\left(7.4 \times 10^{3}+1.4 \times \% P B\right) m_{w}{ }^{3}-\left(8.2 \times 10^{2}-0.3 \times \% P B\right) m_{w}^{4}\right)\left(0.7 / q_{e}^{\prime \prime}\right) \quad 0.0 \leq m_{w} \leq m_{w, c r} \\
& t_{d}=\left.t_{d}\right|_{m_{w, c r}} \\
& t_{i p b}=\left(1.53 \times 10^{2}-1.21 \times \% P B\right)+(-2.44+0.02 \times \% p b) q_{e}^{\prime \prime}
\end{aligned}
$$




$$
\begin{array}{ll}
t_{d}=\left(9.72 \times 10^{-1} / q_{e}^{\prime \prime}\right)\left(-3.02 \times 10^{2}+2.35 \times 10^{3} \times m_{w}\right) & 0.0 \leq m_{w} \leq m_{w, c r} \\
t_{d}=\left(\frac{9.72 \times 10^{-1}}{q_{e}^{\prime \prime}}\right)\left(5.12 \times 10^{3}+2.52 \times 10^{2} \times m_{w}\right) & m_{w}>m_{w, c r} \\
t_{i p b}=\left(6.29 \times 10^{1}-6.79 \times 10^{-1} \times q_{e}^{\prime \prime}\right) . &
\end{array}
$$

As these correlations show there is a significant difference between wood and PMMA behaviours. For both cases the drying time is inversely proportional to the incident heat flux. However, the dependence on the amount of applied water is substantially different. For wood samples the behaviour is strongly non-linear which is a significant departure from the simple correlation proposed by Takahashi [8]. Apriori, two factors may be seem to cause this non-linearity: (i) the non-linear thermal behaviour of the sample during cooling and ignition periods, and (ii) its porous structure. In fact, our experiments with PMMA show that the second factor holds, as the drying time for PMMA demonstrates a linear behaviour with water loading.

Figures 6 and 7 illustrate the correlations of the measured re-ignition times for Radiata pine and PMMA using eqs. (1) to (6). Experimental data for wood were also correlated using Takahashi's formula [8] which can be written as: $t_{r i}=t_{i p b}+\left(m_{w} \times H_{v}\right) / q_{e}^{\prime \prime}$. Here $H_{v}$ represents the latent heat of evaporation for water. Although exact agreement cannot be expected, the magnitude and direction of the trend have been well predicted by the formulation presented here. This in turn, provides a reasonable level of accuracy for both materials. Typically, the maximum difference for wood and PMMA are less than 14\% and $8 \%$, respectively.

As Figure 6 shows, the linear relation outlined by Takahashi also provides some estimation of the re-ignition time. Although this relation predicts the direction of the trend, it does not closely follow the experimental data. As a result, Takahashi's predictions are

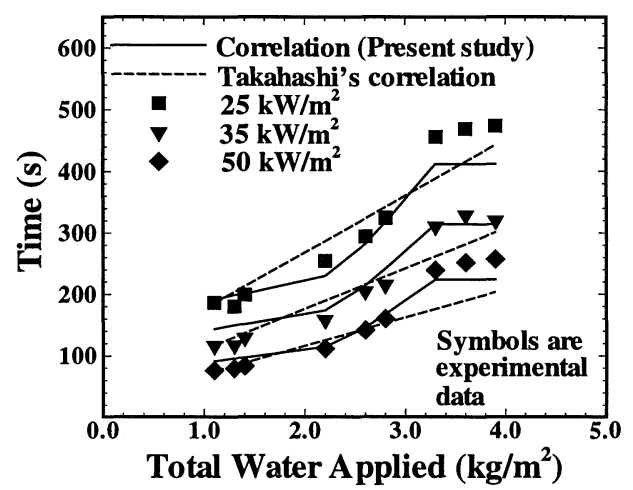

FIGURE 6: Correlation of the re-ignition time for Radiata pine (10\% pre-burn). generally less accurate than those of the present formulation. The worst discrepancy between Takahashi's predictions and measurements occurred for $m_{w}>m_{w, c r}$, where the experimental results were under-predicted by about $25 \%$.

\section{ANALYSIS}

In this section, simulations of the experimental results are presented. For this purpose $t_{d}$ and $t_{i p b}$ are estimated based on sound physical principles. The re-ignition time is then calculated from: $t_{r i}=t_{d}+t_{i p b}$. 


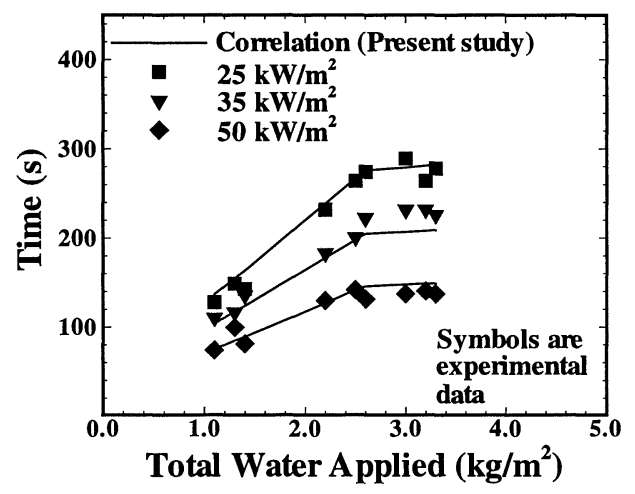

FIGURE 7: Correlation of the re-ignition time for PMMA (10\% pre-burn).

\section{PMMA}

As pointed out before, for the conditions of the present study, external radiation is the main source of heat supply for the evaporation process. Since for the range of heater temperatures used in this study (550$700{ }^{\circ} \mathrm{C}$ ) the absorption coefficient of water is close to unity, it is quite reasonable to assume that all of the radiative energy supplied by the heater is absorbed by the liquid layer. The characteristic drying time $\left(t_{d}{ }^{*}\right)$ for this case can then be expressed as follows:

$$
t_{d}^{*}=\left(m_{w} \times H_{v}\right) / q_{e}^{\prime \prime} .
$$

For PMMA the difference between $t_{d}$ and $t_{d}{ }^{*}$ is small, in fact less than $8 \%$. Hence, $t_{d}$ may be calculated from eq. (7) with a high degree of confidence. For $m_{w}>m_{w, c r}$ it may be assumed that $t_{d}^{*}=\left.t_{d}^{*}\right|_{m w, c r}$.

This greatly simplifies the calculation of $t_{i p b}$. The fact that $t_{d}$ and $t_{d}{ }^{*}$ are very close means that during evaporation, the total heat flux through the exposed surface of the sample must be close to zero. Since the heat transfer process in the solid is governed by the heat conduction equation and the back surface is insulated, the time constant for temperature changes would be $t_{1}=L^{2} / \alpha$, where $L$ and $\alpha$ are the sample thickness and thermal diffusivity, respectively. For a $9 \mathrm{~mm}$ thick PMMA sample this time constant is of the order of 10 minutes.

This time can be compared with a characteristic time of ignition process $\left(t_{i p b}{ }^{*}\right)$ which is written as:

$$
t_{i p b}^{*}=\frac{c_{p} \rho \delta \Delta T}{q_{e}^{\prime \prime}}
$$

where, $c_{p}, \rho, \delta$ and $\Delta T$ are the specific heat of PMMA, density of PMMA, thermal penetration depth and temperature rise, respectively. The average value of $\delta$ which is calculated from: $\delta=\left(\alpha \times t_{d}^{*}\right)^{1 / 2}$ is about $3 \mathrm{~mm}$. The temperature rise may be obtained from the following consideration. As was observed in the experiments, evaporation took place from the upper boundary of the liquid layer. On the solid-liquid interface the temperature for both materials must be the same. The value of this temperature has to be between the initial water temperature and the boiling temperature. Taking into account the ignition temperature of PMMA which is about $540 \mathrm{~K}$, the temperature rise would be in the range of $170-270 \mathrm{~K}$. This gives an estimate of $25 \mathrm{~s}$ for $t_{i p b}{ }^{*}$ at an incident heat flux of $50 \mathrm{kw} / \mathrm{m}^{2}$.

Since $t_{i p p^{*}}{ }^{*}<<t_{1}$, the temperature change during evaporation must be negligible. This means that $t_{i p b}{ }^{*}$ is essentially constant irrespective of previous evaporation history. This result is quite consistent with our experimental data. Therefore, $t_{i p b}$ here is assumed to be constant and it is calculated from eq. (8). 


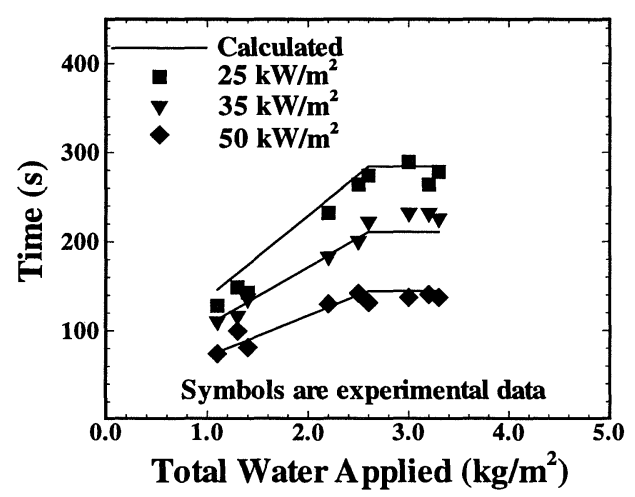

FIGURE 8: A comparison of the measured and calculated re-ignition times for PMMA (10\% pre-burn).
Based on the procedure outlined here re-ignition times for PMMA were calculated and compared with the experimental data. The results are summarised in Figure 8. Clearly, the overall agreement is quite good with a maximum difference of less than $17 \%$. This means our assumptions are reasonable. Generally, the agreement is much better for high heat fluxes $\left(q_{e}^{\prime \prime} \geq 35 \mathrm{~kW} / \mathrm{m}^{2}\right)$.

\section{Wood}

Almost the same arguments may be applied in the case of wood specimens. However, for wood, generally $t_{d}$ is not equal to $t_{d}^{*}$, which means that there is heat exchange between the sample and water during the evaporation period. Therefore, the cooling effect is not negligible. As a result, $t_{i p b}$ may not be taken as a constant value. To model this, a detailed numerical model of wood pyrolysis [13] is used here. This model includes heat release rate, pyrolysis, surface temperature and mass loss rate predictions. Ignition is assumed to occur once the mass flux of volatiles reaches a critical value $\left(m_{c r}{ }^{\prime \prime}\right)$. This value may be obtained from weight loss data. The model takes into account the degree of char formation and the variations in thermal conductivity, specific heat and density with temperature. It also accounts for thermal radiation and convective cooling from the exposed surface. The model, however, assumes that the solid is homogeneous and, hence, neglects the porous structure of wood.

Since the exact value of the heat loss due to cooling (by the water spray) was not known, a sensitivity analysis was performed. The numerical simulation involved 4 phases. These are: (1) ignition, (2) burning, (3) cooling and (4) re-ignition. A typical calculation was started by setting $m_{c r}^{\prime \prime}$ and $q_{e}^{\prime \prime}$ to the desired values. In order to satisfy the ignition criterion, the model was run for a proper duration and it was allowed to continue until a given amount of pre-burn was reached.

The cooling phase was then started by setting the incident heat flux to an assumed value for heat loss due to cooling $\left(q_{c}^{\prime \prime}\right)$. The duration of the cooling phase was assumed to be the same as the drying time and it was obtained from: $t_{d}=\left(m_{w} \times H_{v}\right) /\left(q_{e}^{\prime \prime}+\left|q_{c}^{\prime \prime}\right|\right)$. Both pre-burn and $q_{c}^{\prime \prime}$ were varied systematically, as the controlling parameters of the numerical simulation. The last phase was started by setting the incident heat flux to $q_{e}^{\prime \prime}$. The pyrolysis calculation was then continued until the surface temperature and volatiles mass flux increased to their values at the beginning of the cooling phase.

A typical set of results is presented in Figure 9, where the measured and calculated re-ignition times at an incident heat flux of $25 \mathrm{~kW} / \mathrm{m}^{2}$ are compared. As shown in the figure, the overall behaviour of the experimental data has been well reproduced. However, there are some inconsistencies. In particular, the model provides a linear relationship between the applied water and re-ignition time, whereas, experimental data show a non-linear behaviour. This can be attributed to our assumption about porosity. 


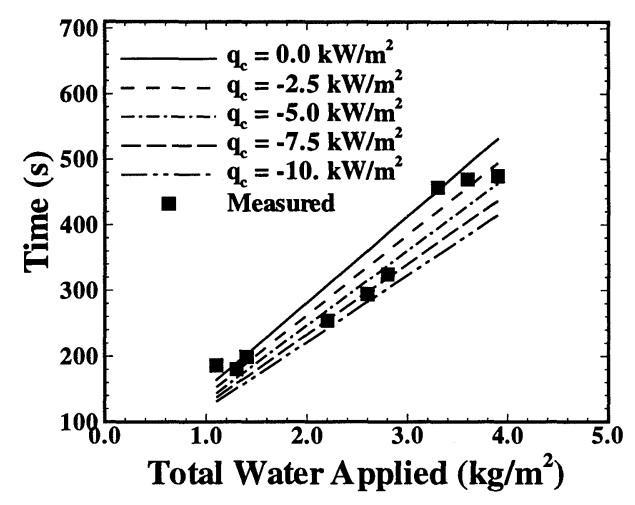

FIGURE 9: A comparison of the measured and calculated re-ignition times for Radiata pine at incident flux of $25 \mathrm{~kW} / \mathrm{m}^{2}$ and $10 \%$ pre-burn.
Based on the sensitivity analysis performed here, the best agreement between predictions and experimental data is obtained for $q_{c}^{\prime \prime}=-5 \mathrm{~kW} / \mathrm{m}^{2}$ with a maximum difference of less than $12 \%$.

\section{CONCLUSIONS}

In this paper the results of a combined experimental and theoretical study of the re-ignition phenomenon of solid fuels are presented. In particular, the effect of a water spray on the overall behaviour of the material is investigated. This study leads to the following conclusions:

(i) The re-ignition phenomenon is greatly affected by the amount of water applied, the incident heat flux and the degree of pre-burn.

(ii) There is a critical amount of applied water beyond which the re-ignition time remains constant. However, this critical value is not unique and depends on the sample size, orientation and geometry.

(iii) The ignition time of partially burnt material is essentially independent of the evaporation history and is small compared with the total re-ignition time. As a result, the re-ignition time is mostly determined by the drying time.

(iv) The dependence of the re-ignition time on water loading is non-linear for wood, in contrast to PMMA. The departure from linearity is determined by mechanical structure (porosity, cracking, etc) of material and water loading only.

(v) The re-ignition of wood and PMMA objects may happen in real fire-fighting situations in a period of time which is shorter than the time required to control the fire. To assess the probability of such events a non-linear correlation is needed for wood. The application of linear correlations may result in overestimation of the reignition time.

(vi) From a theoretical point of view, there is a need for a model that takes into account the effect of porosity on the re-ignition characteristics.

\section{ACKNOWLEDGMENTS}

This work was funded by an Australian Research Council Award in collaboration with the WorkCover Authority of NSW and Wormalds. The authors also gratefully acknowledge the 
assistance of Mr. D. Pearson from the WorkCover Authority of NSW.

\section{REFERENCES}

1. Tamanini, F., "A Study of the Extinguishment of Vertical Wood Slabs in SelfSustained Burning by Water Spray Application", Combustion Science and Technology, 14, 1-15, 1976.

2. Atreya, A., "Effect of Water on Ignition of Cellulosic Materials", Research Proposal, National Bureau of Standards, Washington D.C., USA, 1985.

3. Depew, C.A., Corlett, R.C., Yu, T.I. and Cruz, G.A., "Response of Wood Boards to Radiative Heating and Water Spray", Fall meeting of the Western State Section of the Combustion Institute, El Segundo, Cal, 1973.

4. Usui, K., "Transaction of the Architectural Institute of Japan", 41, 100, 1950.

5. Takahashi, S., "On the Re-ignition", Bulletin of Japanese Association of Fire Science and Engineering, 32:1, 41, 1982.

6. Takahashi, S., "Heat Transfer and Ignition of the Extinguished Wood Residue and the Wet Filter Paper", Report of Fire Research Institute of Japan, 56, 1, 1983.

7. Takahashi, S., "Re-ignition of the Carbonised Wood and the Water Soaked Wood Ember", Report of Fire Research Institute of Japan, 58, 33, 1984.

8. Takahashi, S., "Experiments and Theory in the Extinction of a Wood Crib", Fire Safety Science-Proceedings of the First International Symposium, 1197-1206, 1986.

9. "Standard Test Method for Heat and Visible Smoke Release Rates for Materials and Products Using an Oxygen Consumption Calorimeter (E 1354)", American Society for Testing and Materials, Philadelphia, USA, 1990.

10. Heskestad, G., "The Role of Water in the Suppression of Fires", Journal of Fire Flammability, 12, 254-262, 1980.

11. Nam, S., "Numerical Simulation of Actual Delivered Density of Sprinkler Spray Through Fire Plumes", Atomization and Sprays, 4, 385-404, 1994.

12. Grissom, W.M. and Wierum, F.A., "Liquid Spray Cooling of a Heated Surface", Int. J. Heat Mass Transfer, 24, 261-271, 1981.

13. Novozhilov, V., Moghtaderi, B., Fletcher, D.F. and Kent, J.H., "CFD Modelling of Wood Combustion", Fire Science and Technology-Proceedings of the Second AsiaOceania Symposium, Kabarovsk, Russia, 286-297, 1995. 